\title{
Are (pseudo)bulges in isolated galaxies actually primordial relics?
}

\author{
M. Fernández Lorenzo ${ }^{1}$, J. Sulentic ${ }^{1}$, L. Verdes-Montenegro ${ }^{1}$, J. Blasco-Herrera ${ }^{1}$, M. \\ Argudo-Fernández ${ }^{1}$, J. Garrido ${ }^{1}$, P. Ramírez-Moreta, J. E. Ruiz ${ }^{1}$, S. Sánchez-Expósito ${ }^{1}$ \\ and J.D. Santander-Vela ${ }^{1}$ \\ Instituto de Astrofísica de Andalucía, Granada, Spain \\ mirian@iaa.es
}

\begin{abstract}
We present structural parameters and ( $\mathrm{g}-\mathrm{i}$ ) bulge/disk colors for a large sample (189) of isolated AMIGA galaxies. The structural parameters of bulges were derived from the 2D bulge/disk/bar decomposition of SDSS i-band images using GALFIT. Galaxies were separated between classical bulges $\left(\mathrm{n}_{b}>2.5\right)$ and pseudobulges $\left(\mathrm{n}_{b}<2.5\right)$, resulting in a dominant pseudobulge population $(94 \%)$ with only 12 classical bulges. In the $\left\langle\mu_{e}\right\rangle-R_{e}$ plane, pseudobulges are distributed below the elliptical relation (smaller $R_{e}$ and fainter $\mu_{e}$ ), with the closest region to the Kormendy relation populated by those pseudobulges with larger values of $\mathrm{B} / \mathrm{T}$. We derived (g-i) bulge colors using aperture photometry and find that pseudobulges show median colors $(\mathrm{g}-\mathrm{i})_{b} \sim 1.06$, while their associated disks are much bluer, $(\mathrm{g}-\mathrm{i})_{d} \sim 0.77$. Moreover, $64 \%(113 / 177)$ of pseudobulges follow the red sequence of early-type galaxies. Bluer pseudobulges tend to be located in galaxies with the highest likelihood of tidal perturbation. The red bulge colors and low $\mathrm{B} / \mathrm{T}$ values for AMIGA isolated galaxies are consistent with an early formation epoch and not much subsequent growth. Properties of bulges in isolated galaxies contrast with a picture where pseudobulges grow continuosly via star formation. They also suggest that environment could be playing a role in rejuvenating the pseudobulges.
\end{abstract}

Subject headings: galaxies: general — galaxies: fundamental parameters — galaxies: interactions

\section{Introduction}

Important clues about spiral galaxy formation lie in the nature of their central bulges. There are two main stellar systems called "bulges" in the current literature: A) classical bulges, characterized by an old stellar population, dynamically supported by velocity dispersion, less flat than disks and featureless; B) disk-like or pseudo-bulges (Kormendy \& Illingworth 1982), more flattened and rotationally supported than classical bulges, contain more dust and some show recent star formation (SF) (Kormendy \& Kennicutt 2004). Pseudobulges can contain substructures such as bars, rings and spiral arms. Surface brightness profiles of pseudobulges and classical bulges have Sérsic index (Sérsic 1963) $\mathrm{n}_{b} \leq 2$ and $\mathrm{n}_{b} \geq 2$ respectively with little to no overlap (Fisher \& Drory
2008).

Different scenarios have been proposed for the formation of classical bulges and pseudobulges. Classical bulges are thought to form, as elliptical galaxies, in a rapid and/or violent process. This picture includes both the monolithic collapse (Eggen et al. 1962) and merger scenarios (Bender et al. 1992). However, pseudobulges are thought to grow slowly via redistribution of disk material augmented by gas accretion and other secular processes (see Kormendy \& Kennicutt 2004, for a review). Giant clumps of intense SF have been observed in high redshift spiral galaxies. Gravitational instabilities have been proposed to build bulges via clump migration (Genzel et al. 2008; Bournaud et al. 2014), but the type of bulge remains unclear.

Optical colors of galaxies reflect mainly their 
stellar populations. The distribution of galaxy colors in the $(\mathrm{g}-\mathrm{r})$ vs. $(\mathrm{u}-\mathrm{g})$ plane (Strateva et al. 2001) shows a clear separation into red and blue sequences, corresponding roughly to early- (E, $\mathrm{S} 0$, and $\mathrm{Sa}$ ) and late-type (Sb, Sc, and Irr) galaxies, as expected from the respective dominance of old and young stellar populations. Since pseudobulges are thought to grow from disk material, they presumably preserve some memory of their disky origin. Fisher et al. (2009) found a $\mathrm{SF}$ rate in pseudobulges similar to those found in the disks of their host galaxies (not environmentally selected), interpreting this result to be consistent with pseudobulge stellar mass growth via moderate SF. In this context the stellar population in pseudobulges should be younger and bluer than in classical bulges/elliptical galaxies. The question is, of course, how much bluer? Existing bibliography on bulge colors (Peletier \& Balcells 1996; Gadotti \& Dos Anios 2001; MacArthur et al. 2004; Kannappan et al. 2004; Gadotti 2009), does not target especifically isolated galaxies, which should best reflect their origins because of their minimized environmental evolutionary effects.

This letter presents a study of bulge colors in the AMIGA (Analysis of the interstellar Medium of Isolated GAlaxies, Verdes-Montenegro et al. 2005) sample of galaxies. This sample contains predominantly $(\sim 66 \%)$ late-type spirals with small bulges $(\mathrm{B} / \mathrm{T}<0.1$; Sulentic et al. 2006; Durbala et al. 2008), and represents a fruitful sample to explore colors of bulges for galaxies near their primordial state (minimal merger/accretion/tidal effects). Throughout this article, the concordance cosmology with $\Omega_{\Lambda 0}=0.7, \Omega_{\mathrm{m} 0}=0.3$ and $\mathrm{H}_{0}=70 \mathrm{~km} \mathrm{~s}^{-1} \mathrm{Mpc}^{-1}$ is assumed.

\section{Sample selection}

The AMIGA sample is based on a refinement of the Catalogue of Isolated Galaxies (CIG; Karachentseva 1973), where two complementary isolation parameters were defined to select the most isolated galaxies in the CIG (Verley et al. 2007): the tidal force $\left(\mathrm{Q}_{\text {kar }}\right)$ and the local number density $\left(\eta_{k}\right)$. The isolation parameters have been recently improved for the 636 AMIGA galaxies in the Sloan Digital Sky Survey (hereafter AMIGASDSS; Argudo-Fernández et al. 2013).
We selected 298 spiral $(\mathrm{T}=1-8)$ galaxies from the complete AMIGA-SDSS sample with recession velocities $\mathrm{V}_{r}>1500 \mathrm{~km} / \mathrm{s}($ median $\approx 7000 \mathrm{~km} / \mathrm{s}$ ) and isolation parameters $\mathrm{Q}_{k a r}<-2$ and $\eta_{k}<2.7$, which ensures that the galaxies have been unperturbed by mayor neighbours in the last 5 Gyr. We downloaded the images of these galaxies from the SDSS-III (DR8 Aihara et al. 2011; Blanton et al. 2011) in gri bands. In a few cases two or more frames were combined using the IRAF task imcombine. Nineteen galaxies were rejected because a bad combination of the images (2) or a close saturated star (17). The final sample is composed of 279 isolated spiral galaxies.

\section{Data analysis}

\subsection{Bulge structural parameters}

Total magnitudes were derived using SExtractor (Bertin \& Arnouts 1996) in the gri bands. The magnitudes were corrected applying the Galactic dust extinction computed by SDSS following Schlegel et al. (1998), and the k-correction computed using the code kcorrect (Blanton \& Roweis 2007).

Structural modeling of galaxy bulges was performed using the GALFIT package (Peng et al. 2010) applied to the i-band SDSS images. We fit a Sérsic function to the bulges and an exponential function to the disks. We identified visually galaxies hosting a bar using the i-band and color images of SDSS and in these cases we fit an additional Sérsic function for this component. In all cases, the model was convolved with a pointspread function (PSF) generated from the SDSS psField.

Since the fit depends strongly in the input values, we fitted each galaxy with a variety of initial values, inspecting the results and accepting a fit if the median of the residuals in the $5 \times 5$ innermost pixels was lower than $10 \%$. This was achieved for 189 galaxies, which form our sample hereinafter (45\% barred). Four galaxies (the three Sdm and one $\mathrm{Sd}$ ) show no bulge. Eight galaxies show signs of interaction and their disks could not be properly fitted. Four galaxies are affected by a star in the central part and 24 (most of them highly inclined) show dust lines in their centers which confuse the GALFIT bulge fit. Finally, 50 galaxies present residuals larger than the $10 \%$ of the flux in 

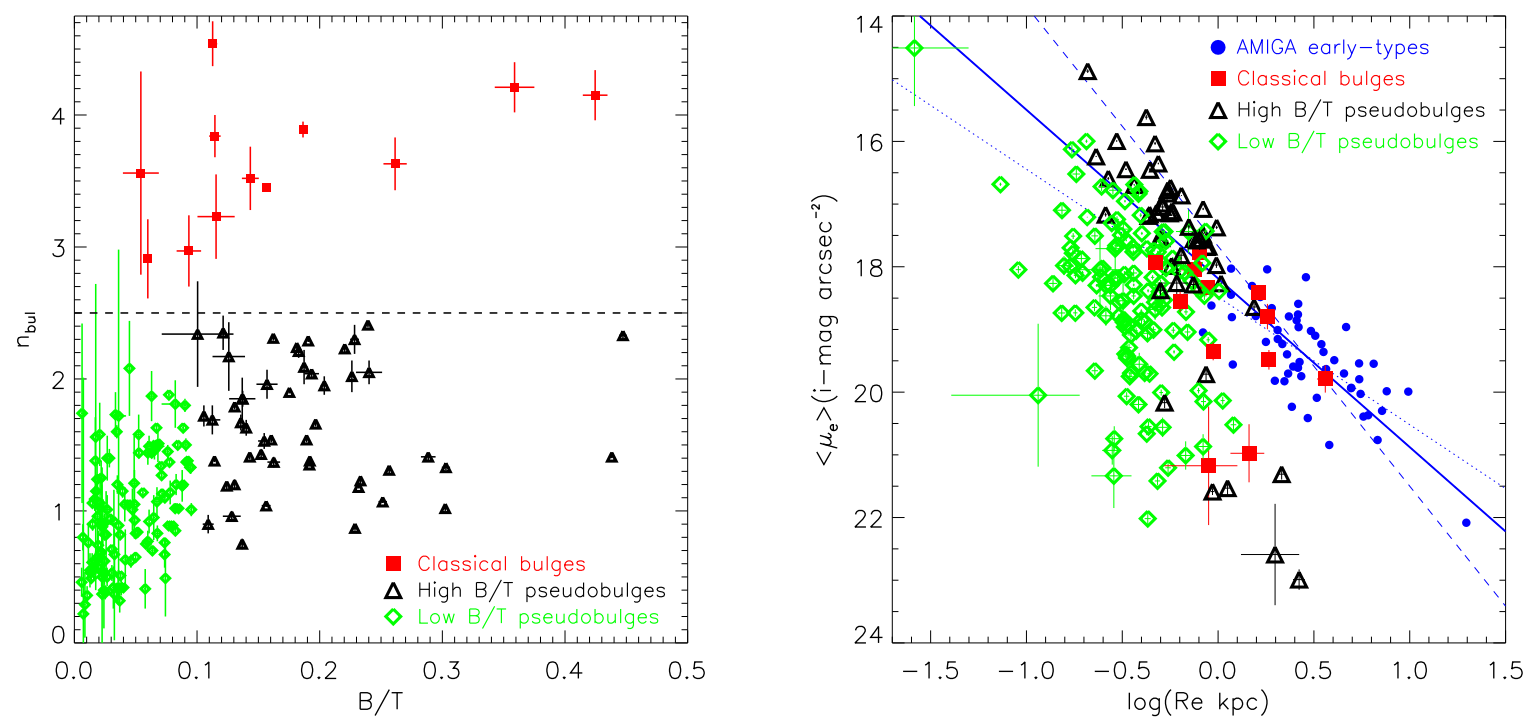

Fig. 1.- Structural parameters in the i-band for the bulges in the AMIGA-SDSS sample. Left panel: bulge Sérsic index versus bulge-to-total ratio. Right panel: the Kormendy relation for classical bulges (red squares), low-B/T pseudobulges (green diamonds), and high-B/T pseudobulges (black triangles) according to the left panel. As comparison, the Kormendy relation for the AMIGA early-type galaxies is represented (blue points). A ordinary least square Y vs. X (points line), X vs. Y (dashed line) and bisector fit (solid line) to these galaxies are shown.

the innermost region, mostly caused by unreliable parameters in the fit that could not be solved with any set of initial parameters tested. In the g and $r$ bands, the fits were done using the i-band parameters leaving free the magnitudes of the components and the disk scale lenght. We compare this metodology with the free fit in $\mathrm{g}$ and $\mathrm{r}$-bands. The parameters obtained for the disk are very stable, with a difference in magnitude lower than 0.01 for $80 \%$ of the cases. Differences in bulge parameters are larger although classification based in Sérsic index (see below) would change for only $5 \%$ of the galaxies. Further discussion and details will be presented in a follow up paper.

Gadotti (2008) showed that the structural properties of bulges can be reliably retrieved when $\mathrm{a}_{e}$ is larger than $\sim 80 \%$ of the PSF half width at half maximum (HWHM). We checked the ratio between our $\mathrm{a}_{e}$ (which is PSF corrected) and the seeing HWHM given by SDSS for each iband image. Only 6 galaxies $(3 \%$ of the sample) have $a_{e} / H W H M<0.8$ and 14 galaxies have $\mathrm{a}_{e} / \mathrm{HWHM}<1$.
GALFIT provides the effective radius along the semimajor axis $\left(\mathrm{a}_{\mathrm{e}}\right)$. To calculate the circular effective radius, we used $\mathrm{R}_{\mathrm{e}}=\mathrm{a}_{\mathrm{e}} \sqrt{(b / a)_{b}}$, where $(b / a)_{b}$ is the bulge axis ratio. We derive the average surface brightness within the effective radius using equation 1 (Graham \& Driver 2005):

$$
<\mu_{e}>=m_{i}+2.5 \log \left(2 \pi R_{e}^{2}\right)
$$

where $\mathrm{m}_{i}$ is the magnitude in the $\mathrm{i}$-band given by GALFIT for the bulge component, corrected by Galactic extinction and k-correction. $\left\langle\mu_{e}\right\rangle$ was corrected for cosmological dimming multiplying the total flux by $(1+\mathrm{z})^{4}$, where $\mathrm{z}$ is de redshift of the galaxy.

The bulge parameters obtained from the GALFIT fit are represented in Fig. 11 The left panel presents the bulge Sérsic index versus the bulgeto-total $(\mathrm{B} / \mathrm{T})$ ratio. We used $\mathrm{n}_{b}$ to separate classical bulges $\left(\mathrm{n}_{b}>2.5\right)$ and pseudobulges $\left(\mathrm{n}_{b}<2.5\right)$. We found that $94 \%$ of bulges in the AMIGA sample are classified as pseudobulges. We used different symbols for high-B/T $(\mathrm{B} / \mathrm{T}>0.1)$ and low $-\mathrm{B} / \mathrm{T}(\mathrm{B} / \mathrm{T}<0.1)$ pseudobulges in the Figures 
TABLE 1

DERIVED DATA FOR THE AMIGA-SDSS SAMPLE.

\begin{tabular}{|c|c|c|c|c|c|c|c|c|c|c|c|c|c|c|}
\hline $\begin{array}{l}\text { CIG } \\
\text { (1) }\end{array}$ & $\begin{array}{l}\mathrm{B} / \mathrm{T} \\
(2)\end{array}$ & $\begin{array}{c}\mathbf{m}_{i, d} \\
(\mathrm{mag}) \\
(3)\end{array}$ & $\begin{array}{c}\mathbf{h}_{R} \\
(\operatorname{arcsec}) \\
(4)\end{array}$ & $\begin{array}{c}(\mathbf{b} / \mathbf{a})_{d} \\
(5)\end{array}$ & $\begin{array}{c}\mathbf{m}_{i, b} \\
(\mathrm{mag}) \\
(\mathbf{6})\end{array}$ & $\begin{array}{c}\mathbf{a}_{e} \\
(\operatorname{arcsec}) \\
(7)\end{array}$ & $\begin{array}{l}\mathbf{n}_{b} \\
(8)\end{array}$ & $\begin{array}{c}(\mathbf{b} / \mathbf{a})_{b} \\
(\mathbf{9})\end{array}$ & $\begin{array}{c}\mathbf{m}_{i, b a r} \\
(\mathrm{mag}) \\
(\mathbf{1 0})\end{array}$ & $\begin{array}{c}<\mu_{e}(\mathbf{i}-\text { band })> \\
\left(\operatorname{mag} / \operatorname{arcsec}^{2}\right) \\
(\mathbf{1 1})\end{array}$ & $\begin{array}{c}\mathbf{R}_{e} \\
(\mathrm{kpc}) \\
(\mathbf{1 2})\end{array}$ & $\begin{array}{l}\mathbf{M}_{i} \\
\mathrm{mag} \\
(\mathbf{1 3})\end{array}$ & $\begin{array}{c}(\mathrm{g}-\mathbf{i})_{c, d} \\
(\mathbf{1 4})\end{array}$ & $\begin{array}{c}(\mathrm{g}-\mathbf{i})_{c, b} \\
(\mathbf{1 5})\end{array}$ \\
\hline 1 & 0.01 & 12.75 & 10.72 & 0.52 & 17.33 & 0.99 & 0.61 & 0.56 & & 18.47 & 0.36 & -22.35 & 0.87 & 1.16 \\
\hline 2 & 0.03 & 14.29 & 8.06 & 0.63 & 18.00 & 1.88 & 0.53 & 0.15 & 17.77 & 19.09 & 0.35 & -20.83 & 0.70 & 0.91 \\
\hline 4 & 0.01 & 11.15 & 25.16 & 0.27 & 15.79 & 1.49 & 0.49 & 0.48 & & 17.70 & 0.17 & -21.49 & 1.08 & 1.32 \\
\hline 7 & 0.07 & 13.98 & 6.75 & 0.73 & 16.80 & 0.51 & 1.07 & 0.79 & 17.73 & 16.84 & 0.38 & -22.45 & 0.89 & 1.17 \\
\hline 9 & 0.04 & 14.16 & 9.14 & 0.28 & 17.66 & 1.12 & 0.42 & 0.52 & & 19.00 & 0.46 & -21.25 & 0.65 & 0.98 \\
\hline 29 & 0.24 & 15.12 & 15.03 & 0.15 & 16.37 & 10.81 & 2.05 & 0.70 & & 22.99 & 2.65 & -19.05 & 0.63 & 0.70 \\
\hline 33 & 0.02 & 12.64 & 9.15 & 0.60 & 16.83 & 0.89 & 0.57 & 0.85 & 15.75 & 18.30 & 0.23 & -21.21 & 0.83 & 1.08 \\
\hline 39 & 0.07 & 13.64 & 8.99 & 0.45 & 16.53 & 2.99 & 1.46 & 0.83 & & 20.56 & 0.51 & -19.45 & 0.66 & 0.98 \\
\hline 40 & 0.09 & 14.47 & 12.99 & 0.32 & 16.47 & 1.42 & 2.97 & 0.45 & 15.14 & 18.05 & 0.76 & -22.27 & 0.86 & 1.21 \\
\hline 49 & 0.01 & 12.82 & 15.18 & 0.71 & 17.30 & 0.92 & 0.90 & 0.86 & 15.71 & 18.79 & 0.30 & -21.57 & 0.74 & 1.09 \\
\hline.. & .. & .. & .. & .. & .. &.. & .. & .. &.. & .. & .. & .. & .. & .. \\
\hline
\end{tabular}

1 Table 1 is published in its entirety in the machine-readable version and is available at http://amiga.iaa.es A portion is shown here for guidance regarding its form and content. Columns correspond to (1): galaxy identification according to CIG catalog; (2): bulge-to-total luminosity ratio; (3): GALFIT disk magnitude; (4) disk scalelength along the semimajor axis; (5): disk semiaxes ratio; (6): GALFIT bulge magnitude; (7): bulge effective radius along the semimajor axis; (8): bulge Sèrsic index; (9): bulge semiaxes ratio; (10): GALFIT bar magnitude; (11): bulge average surface brightness within the effective radius; (12): bulge effective radius; (13) total absolute magnitude in the i-band; (14) corrected disk (g-i) color; (15) corrected bulge ( $\mathrm{g}-\mathrm{i}$ ) color.

The full table contains additional data on fits and corrections.

to check if the former represent more evolved pseudobulges. In the right panel of Fig. 1 we show the Kormendy relation (Kormendy 1977) between $\left\langle\mu_{e}\right\rangle$ and $\mathrm{R}_{\mathrm{e}}$ for the bulges. As comparison, we represent the Kormendy relation for those early-type galaxies $(\mathrm{T}<0)$ in the AMIGA sample that were fitted with a Sérsic function in Fernández Lorenzo et al. (2013). To derive $\left\langle\mu_{e}>\right.$, we apply the same procedure and corrections used for the bulges but $\mathrm{m}_{i}$ is the total magnitude of the galaxy in the $\mathrm{i}-$ band.

Classical bulges are expected to form an unbroken sequence with the distribution of early-type galaxies in the $\left\langle\mu_{e}\right\rangle-\mathrm{R}_{\mathrm{e}}$ plane (Carollo 1999). Pseudobulges show significantly lower $\left\langle\mu_{e}\right\rangle$ than classical bulges for a given $\mathrm{R}_{\mathrm{e}}$ (Gadotti 2009; Fisher \& Drorv 2010). The right panel of Fig. 1 shows results consistent with these expectations. High-B/T pseudobulges are at the top of the pseudobulges distribution, in a region consistent with the Kormendy relation, which suggests some similarity with classical bulges. Fisher et al. (2009) found a class of non-star forming pseudobulges which are consistent with the parameter correlation of classical bulges and early-type galaxies (Fisher \& Drory 2010). These inactive pseudobulges may be composite systems where both classical and pseudobulge components have similar luminosities. This could be also the case for the high-B/T pseudobulges in our sample. Moreover, some classical and pseudobulges may also be composite systems where one component is more prominent than the other (Peletier et al. 2007; MacArthur et al. 2009).

\subsection{Bulge stellar populations}

We use $(\mathrm{g}-\mathrm{i})$ colors as indicative of the stellar population for bulges and disks in our sample. We used magnitudes from GALFIT to estimate disk colors because the disk fits were very stable (see Sect. 3.1). In the case of bulges, the magnitudes derived from GALFIT for half of the sample resulted in colors that are more than $3 \sigma$ redder than the red sequence fitted for early-type galaxies, and hence these colors are unreliable. This happens especially for later type spirals where the disk contribution is stronger. These anomalous bulge colors might be connected to a change in the disk properties inside the bulge affecting the luminosities obtained from the bulge/disk decomposition. This will be discussed in a subsequent paper. These results motivated us to derive bulge colors using aperture photometry instead. We calculated i-band aperture magnitudes for the galax- 


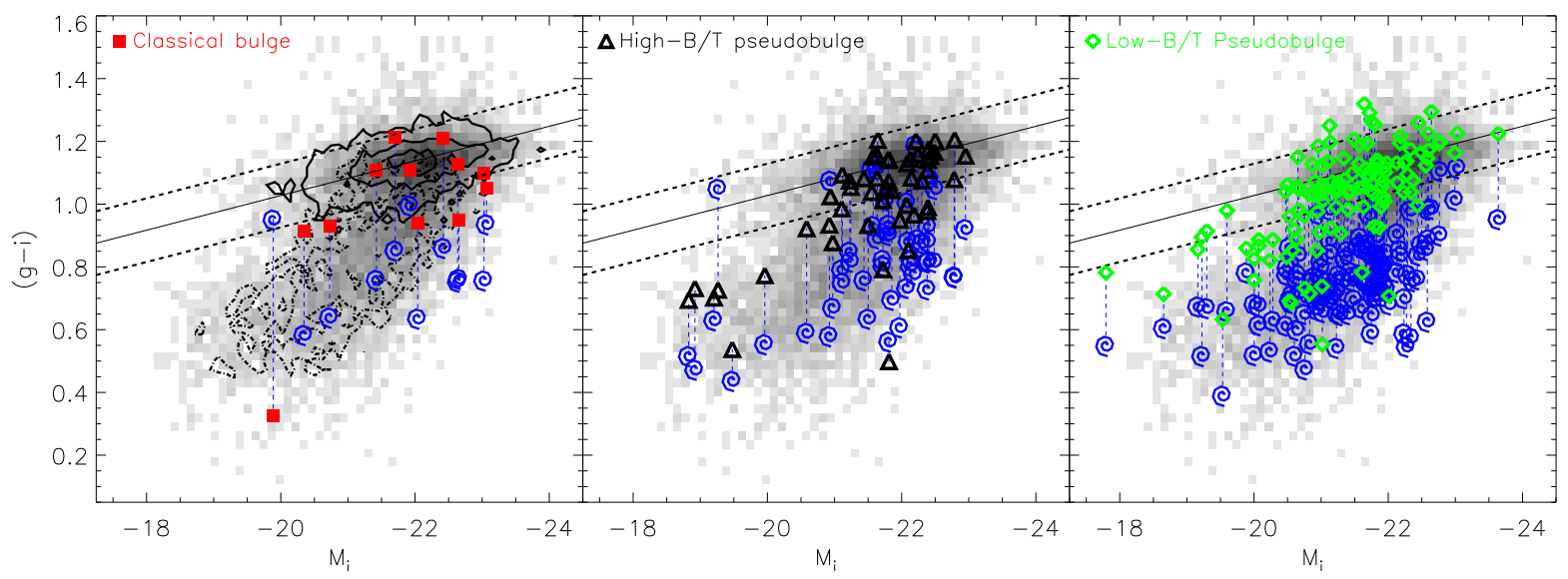

Fig. 2.- $(\mathrm{g}-\mathrm{i})$ color for bulges and the disks (blue spirals) versus the absolute magnitude in the $\mathrm{i}-$ band of the host galaxy $\left(\operatorname{Mag}_{i}\right)$. Different bulge types are represented with different symbols following Fig. 1, The grey-scale represents the density diagram obtained from the Nair \& Abraham (2010) sample at $0.01<z<0.05$. The solid and dashed lines are the linear fit and its $2 \sigma$ for the early-type galaxies. In the first panel, we represent the contours of equal density for these galaxies (solid) and for Sbc-Sd spirals (point-dash).

ies using the IRAF task ELLIPSE, with fits forcing the same center for all the isophotal apertures. We then calculated the aperture magnitudes in the $\mathrm{g}$ and $\mathrm{r}$-bands, fixing the ellipticity and position angle of each isophotal aperture equal to the $\mathrm{i}-$ band values. The bulge is usually defined as the excess inner light over that from the disk. Using our bulge/disk/bar decomposition, we calculate the radius where $\mu($ bulge $+\operatorname{disk}[+$ bar $])-$ $\mu(\operatorname{disk}[+\mathrm{bar}])=-0.1$ and adopt the aperture at this radius for calculating the bulge color. This aperture is smaller than 5 pixels for $15 \%$ of the galaxies. In these cases, it was set to 5 pixels to ensure a minimum loss of bulge flux because of the seeing (width of the SDSS PSF at $1 \%$ level is $\sim 10$ pixels).

In order to compute rest-frame colors, the magnitudes were corrected for Galactic extinction and $\mathrm{k}$-correction (see Sect.3.1). We derived new kcorrection for the bulge using the aperture magnitudes (the global $\mathrm{k}$-corrections results in a difference in the $(\mathrm{g}-\mathrm{i})_{b}$ color lower than 0.02 for $97 \%$ of the bulges). Colors were corrected for the dependence of internal extinction with inclination following Masters et al. (2010, Eq. 3), but only for the Sa-Sc galaxies (87\%) since no dependence in the color with inclination was found in Fernández Lorenzo et al. (2012) for later types. The same correction has been applied to bulge and disk. The structural parameters and colors calcu- lated in this work are listed in Table 1.

In Fig. 2, we present (g-i) color obtained for bulges and disks versus total i-band magnitude. We present as a comparison sample the density diagram obtained from the Nair \& Abraham (2010) sample at $0.01<\mathrm{z}<0.05$ (DR8) (Fernández Lorenzo et al. 2012). We find $63 \%(119 / 189)$ of the bulges within $2 \sigma$ of the red sequence fitted to $\mathrm{E} / \mathrm{S} 0$ galaxies of Nair \& Abraham (2010) sample. Splitting by types, we find $64 \%(113 / 177)$ of pseudobulges as red as early-type galaxies, with $66 \%$ of low-B/T (84/127) and 58\% (29/50) of high-B/T pseudobulges located in the red sequence. According to the Kolmogorov-Smirnov test, the distributions of colors for low-B/T and high-B/T pseudobulges are statistically equal (probability $\mathrm{p}(\mathrm{K}-$ $\mathrm{S})=0.88)$, with a median $(\mathrm{g}-\mathrm{i})$ value of $1.06 \pm 0.11$ and $1.07 \pm 0.13$ respectively. However, the distributions of colors for their associated disks are statistically different $(\mathrm{p}(\mathrm{K}-\mathrm{S})=0.003)$, probably because high- $\mathrm{B} / \mathrm{T}$ pseudobulges are located in earlier spiral types. The mean difference between bulge and disk colors is larger for low $-\mathrm{B} / \mathrm{T}$ than for high-B/T pseudobulges which likely reflects a redder disk (median $(\mathrm{g}-\mathrm{i})_{d}=0.82 \pm 0.14$ versus $0.75 \pm 0.10)$. We found no difference between colors of classical bulges and pseudobulges $\left(\mathrm{p}\left(\mathrm{K}_{-}\right.\right.$ $\mathrm{S})=0.90$ ). This result contrasts with Gadotti (2009), who concludes that pseudobulges were 0.2 


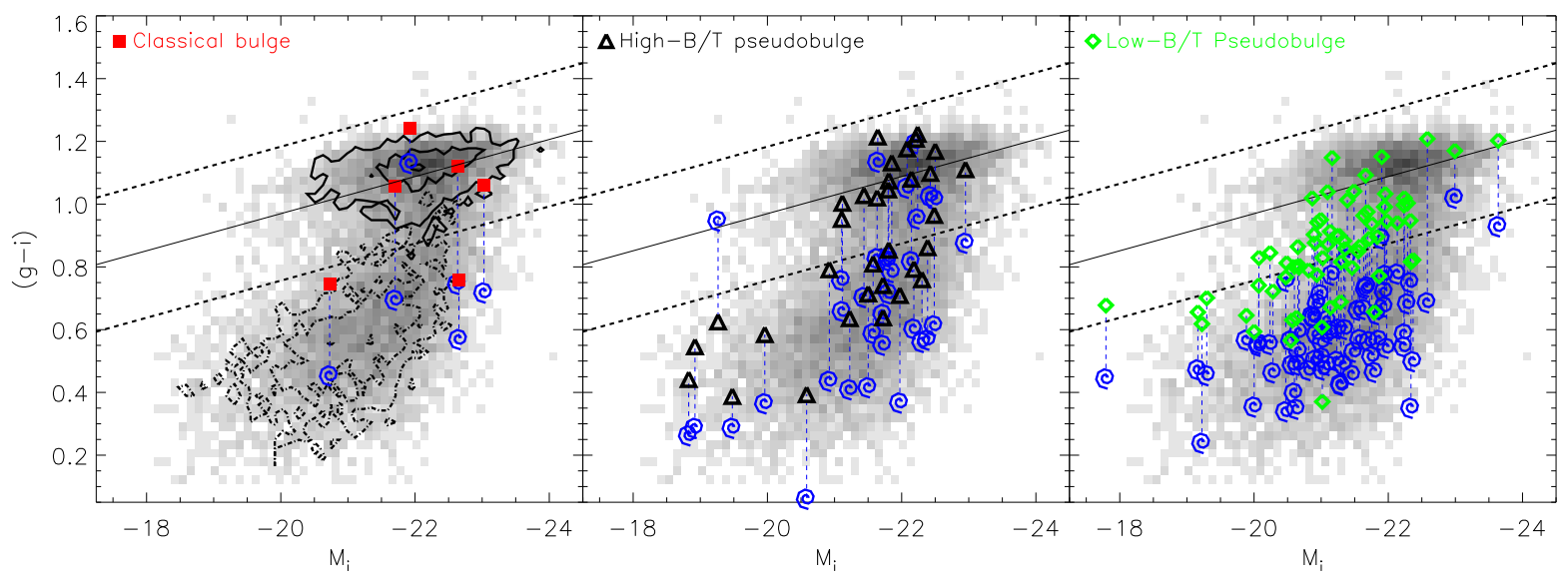

Fig. 3.- (g-i) color for bulges and disks, corrected for internal reddening, versus $\mathrm{Mag}_{i}$. Symbols are the same that in Fig. 2.

mag bluer than classical bulges.

The colors above are corrected only for the differential reddening due to inclination. To check if a full reddening correction could affect the results we used the extinction Av derived from the starlight fit to the stellar continuous (Cid Fernandes et al. 2005, 2009) for those galaxies with SDSS spectra. This is possible for $108 / 189$ of our galaxies and $8811 / 8879$ of the Nair \& Abraham (2010) sample. We used the Calzetti et al. (2000) law and $\mathrm{Rv}=3.1$ to calculate the reddening in the $(\mathrm{g}-\mathrm{i})$ color. In Fig. 3, we reproduce Fig. 2 with colors corrected for internal reddening. The scatter in the red sequence fitted to the E/S0 galaxies from the Nair \& Abraham (2010) sample increases a factor 2 when reddening is taken into account, while the red sequence and the blue cloud are better separated. $60 \%$ of our pseudobulges are still located in the red sequence although an important fraction of low-B/T pseudobulges seem to populate the region between the red sequence and the blue cloud, where early-type spirals and lenticulars are located.

The bulge-disk color differences found here suggest an early formation for most bulges and a different SF history for both components. Pseudobulges in the red sequence seem to follow the same color-magnitude relation than early-type galaxies, i.e., they are redder for brighter host galaxies. Also, the bluer bulges are located in the faintest spiral galaxies. These results could be indicative of a "downsizing" scenario where the most massive galaxies (and their bulges) are the first to be assembled (Cimatti et al. 2006).

\section{Discussion}

An important fraction of pseudobulges in isolated galaxies are as red as early-type galaxies. Due to the degeneracy between age and metallicity affecting the optical colors, is difficult to infer the age of this population. However, the analysis of SDSS fiber spectra for 75 AMIGA galaxies selected from Durbala et al. (2008) showed predominantly old stellar populations ( $10 \mathrm{Gyr})$ and thus anticipated our result (Zhao 2012).

Formation and evolution of pseudobulges are atributted to secular processes (Kormendy \& Kennicutt 2004). The young stars (Peletier \& Balcells 1996; Gadotti \& Dos Anjos 2001; MacArthur et al. 2009) and the SF found inside some pseudobulges (Fisher et al. 2009) point to this ongoing evolution. In Fisher et al. (2009), the specific SFR of some pseudobulges is larger than the disk specific SFR, suggesting an increase of $\mathrm{B} / \mathrm{T}$ through continuous SF.

To check whether a bulge formed through continuous SF can have a color as red as the ones observed here, we performed the following test. We did two simulations with Starburst99 (Leitherer et al. 1999), using an initial mass function of Kroupa and a metallicity of $Z=0.008: 1$ ) an instantaneous burst of $1.5 \times 10^{9} \mathrm{M} \odot$ which evolves 


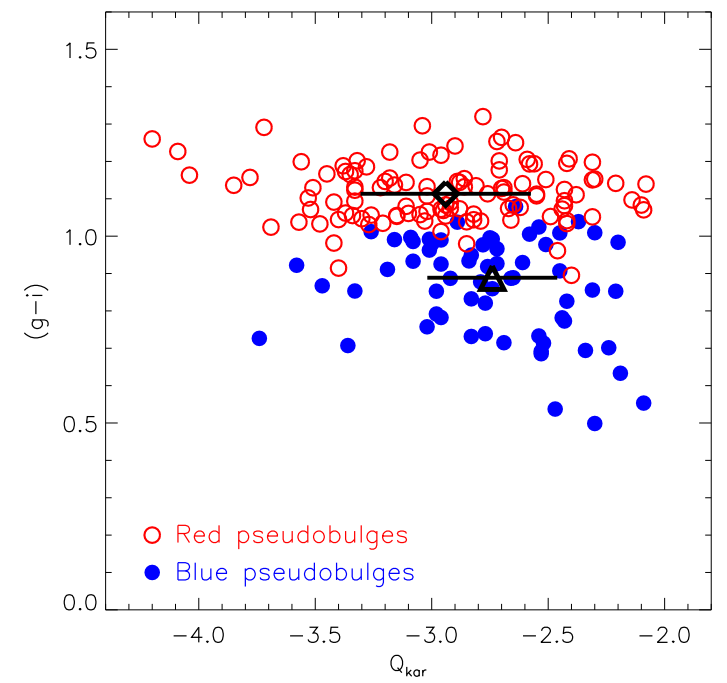

Fig. 4. - ( $\mathrm{g}-\mathrm{i})$ pseudobulge color versus the tidal strength in our sample. Red open circles and blue full circles represent the pseudobulges above and below the red sequence minus $2 \sigma$ fitted in Fig. 2 , The median values and absolute standard deviation in $\mathrm{Q}_{k a r}$ for red (diamond) and blue (triangle) pseudobulges are shown.

along 8 Gyr; 2) a population which grows through a constant SF of $0.2 \mathrm{M} \odot \mathrm{yr}^{-1}$ (mean stellar mass of our bulges is $3 \times 10^{9} \mathrm{M} \odot$ ). The result after 8 Gyr is:

- Instantaneous burst: $(\mathrm{g}-\mathrm{i})=1.13$

- Continous SF: $(\mathrm{g}-\mathrm{i})=0.49$

- Combined model: $(\mathrm{g}-\mathrm{i})=0.59$

Based on this, a bulge formed through continuous $\mathrm{SF}$ should have a very blue $(\mathrm{g}-\mathrm{i})$ color. But even if half of the mass is formed in an instantaneous burst $8 \mathrm{Gyr}$ ago, the blue emission from the continuous SF dominates the color, which is also very blue in the combined model. This is still true if we assume a reddening of 0.2 in $(\mathrm{g}-\mathrm{i})$, the mean value for our bulges.

Gravitational instabilities Genzel et al. 2008; Bournaud et al. 2014), early secular evolution without much subsequent activity (Kormendy \& Kennicut 2004) or a combination of different phenomena could form pseudobulges at an early epoch. However, Fisher et al. (2009) found strongh SF in some pseudobulges and some isolated galaxies also show blue pseudobulges colors. Could the environment play a role in rejuvenating the pseudobulges? Fig. 4 shows pseudobulge color versus tidal strenght $\mathrm{Q}_{\text {kar }}$. We separated red from blue pseudobulges using as boundary the red sequence fitted in Fig. 2 minus $2 \sigma$. The distributions of $\mathrm{Q}_{k a r}$ for red and blue pseudobulges are statistically different $(\mathrm{p}(\mathrm{K}-\mathrm{S})=0.008)$, with a median $\mathrm{Q}_{k a r}$ value of $-2.94 \pm 0.37$ and $-2.74 \pm 0.28$ respectively. Galaxies with red pseudobulges cover the entire range of $\mathrm{Q}_{k a r}$, while bluer pseudobulges show some preference for galaxies with larger $\mathrm{Q}_{k a r}$ (higher crosssection for environmental perturbation). This result is in agreement with Kannappan et al. (2004), who found that galaxies with bluer bulge colors than their disks were connected with morphological peculiarities suggestive of tidal encounters.

\section{Conclusions}

We have performed a 2D bulge/disk/bar decomposition for a sample of 189 isolated galaxies. We used the Sérsic index to separate between classical bulges $\left(\mathrm{n}_{b}>2.5\right)$ and pseudobulges $\left(\mathrm{n}_{b}<2.5\right)$. We found 12 classical bulges and 177 pseudobulges in our sample. Our pseudobulges fall below the $<\mu_{e}>-\mathrm{R}_{\mathrm{e}}$ plane of early-type galaxies, i.e. they are less dense, with the region closest to the Kormendy relation populated by those pseudobulges with larger values of $\mathrm{B} / \mathrm{T}$.

We derived the $(\mathrm{g}-\mathrm{i})$ colors of our bulges using aperture photometry. We found that a $64 \%$ of our pseudobulges follow the red sequence of early-type galaxies: they present colors similar to those presented by early-type galaxies as luminous as their host galaxies (redder for brighter galaxies). These red colors suggest a predominant old stellar population. The bluer bulges in our sample tend to be located in those galaxies more affected by the tidal interactions. The properties of the majority of bulges in isolated galaxies suggest that pseudobulges formed most of their mass in an early epoch, and that specific environmental events could rejuvenate the pseudobulges. In our sample of isolated galaxies, these events were minimized, which would explain our large fraction of late-types and he red colors of their (pseudo)bulges.

We thank the referee for his/her interest in the 
paper and constructive reading and suggestions. This work has been supported by Grant AYA201130491-C02-01 and the Junta de Andalucía (Spain) P08-FQM-4205 and TIC-114.

Funding for SDSS-III has been provided by the Alfred P. Sloan Foundation, the Participating Institutions, the National Science Foundation, and the U.S. Department of Energy. The SDSS-III web site is http://www.sdss3.org/.

\section{REFERENCES}

Aihara, H., et al. 2011, ApJS, 193, 29

Argudo-Fernández, M., Verley, S., Bergond, G., et al. 2013, A\&A, 560, A9

Bender, R., Burstein, D., \& Faber, S. M. 1992, ApJ, 399, 462B

Bertin, E., \& Arnouts, S. 1996, A\&AS, 117, 393

Blanton, M. R., \& Roweis, S. 2007, AJ, 133, 734

Blanton, M. R., Kazin, E., Muna, D., Weaver, B. A., \& Price-Whelan, A. 2011, AJ, 142, 31

Bournaud, F., Perret, V., Renaud, F., et al. 2014, ApJ, 780, 57

Calzetti, D., Armus, L., Bohlin, R. C., et al. 2000, ApJ, 533, 682C

Carollo, C. M. 1999, ApJ, 523, 566

Cid Fernandes, R., Mateus, A., Sodré, L., Stasińska, G., \& Gomes, J. M. 2005, MNRAS, $358,363 \mathrm{C}$

Cid Fernandes, R., Schoenell, W., Gomes, J. M., et al. 2009, Revista Mexicana de Astronomia y Astrofisica Conference Series, 35, 127

Cimatti, A., Daddi, E., \& Renzini, A. 2006, A\&A, 453, L29

Durbala, A., Sulentic, J. W., Buta, R., \& VerdesMontenegro, L. 2008, MNRAS, 390, 881

Eggen, O. J., Lynden-Bell, D., \& Sandage, A. R. 1962, ApJ, 136, 748E

Fernández Lorenzo, M., Sulentic, J., VerdesMontenegro, L., et al. 2012, A\&A, 540, A47
Fernández Lorenzo, M., Sulentic, J., VerdesMontenegro, L., \& Argudo-Fernández, M. 2013, MNRAS, 434, 325

Fisher, D. B., \& Drory, N. 2008, AJ, 136, 773

Fisher, D. B., Drory, N., \& Fabricius, M. H. 2009, ApJ, 697, 630

Fisher, D. B., \& Drory, N. 2010, ApJ, 716, 942

Gadotti, D. A., \& Dos Anjos, S. 2001, Galaxy Disks and Disk Galaxies, 230, 237

Gadotti, D. A. 2008, MNRAS, 384, 420

Gadotti, D. A. 2009, MNRAS, 393, 1531

Genzel, R., Burkert, A., Bouché, N., et al. 2008, ApJ, 687, 59

Graham, A. W., \& Driver, S. P. 2005, PASA, 22, $118 \mathrm{G}$

Kannappan, S. J., Jansen, R. A., \& Barton, E. J. 2004, AJ, 127, 1371

Karachentseva, V. E. 1973, Astrofizicheskie Issledovaniia Izvestiya Spetsial'noj Astrofizicheskoj Observatorii, 8, 3

Kormendy, J. 1977, ApJ, 218, 333

Kormendy, J., \& Illingworth, G. 1982, ApJ, 256, 460

Kormendy, J., \& Kennicutt, R. C., Jr. 2004, ARA\&A, 42, 603K

Leitherer, C., Schaerer, D., Goldader, J. D., et al. 1999, ApJS, 123, 3

MacArthur, L. A., Courteau, S., Bell, E., \& Holtzman, J. A. 2004, ApJS, 152, 175

MacArthur, L. A., González, J. J., \& Courteau, S. 2009, MNRAS, 395, 28

Masters, K. L., et al. 2010, MNRAS, 404, 792

Nair, P. B., \& Abraham, R. G. 2010, ApJS, 186, 427

Peletier, R. F., \& Balcells, M. 1996, AJ, 111, 2238

Peletier, R. F., Falcón-Barroso, J., Bacon, R., et al. 2007, MNRAS, 379, 445 
Peng, C. Y., Ho, L. C., Impey, C. D., \& Rix, H.-W. 2010, AJ, 139, 2097

Schlegel, D. J., Finkbeiner, D. P., \& Davis, M. 1998, ApJ, 500, 525

Sérsic, J. L. 1963, Boletin de la Asociacion Argentina de Astronomia La Plata Argentina, 6, 41

Strateva, I., et al. 2001, AJ, 122, 1861

Sulentic, J. W., et al. 2006, A\&A, 449, 937

Verdes-Montenegro, L., Sulentic, J., Lisenfeld, U., Leon, S., Espada, D., Garcia, E., Sabater, J., \& Verley, S. 2005, A\&A, 436, 443

Verley, S., et al. 2007, A\&A, 472, 121

Zhao, Y. 2012, Ap\&SS, 337, 719

This 2-column preprint was prepared with the AAS LATEX macros v5.2. 